\title{
TWO NEW EXTREMAL PROPERTIES OF THE KOEBE-FUNCTION ${ }^{1}$
}

\author{
R. KLOUTH AND K.-J. WIRTHS
}

\begin{abstract}
Using essentially Löwner's method the extremality of the Koebe-functions with respect to two coefficient problems for inverses of univalent functions is proved.
\end{abstract}

Let $D=\{z|| z \mid<1\}$ and $S=\left\{f \mid f\right.$ regular and univalent in $D, f(0)=f^{\prime}(0)-1$ $=0\}$. K. Löwner [4] proved: If $F(w)=w+\sum_{n=2}^{\infty} A_{n} w^{n}$ is the inverse of a function in $S$, then

$$
\left|A_{n}\right|<(2 n) ! / n !(n+1) !
$$

with equality only for the inverses of the Koebe-functions $k_{o}(z)=z(1+\sigma z)^{-2}$, $|\sigma|=1$.

In this note we shall prove similar results for the functions

$$
\ln F^{\prime}(w), \quad \Delta(F(w), w):=\left(F^{\prime \prime} / F^{\prime}\right)^{\prime}-\frac{1}{2}\left(F^{\prime \prime} / F^{\prime}\right)^{2} .
$$

This work was stimulated by a conjecture of the first author (see [2] and [3]) and the preprint [6] of a lecture given by G. Schober at the Durham Conference on Aspects of Contemporary Complex Analysis in 1979.

THEOREM. Let $F$ be the inverse of a function in $S, K_{1}(w)=k_{1}^{-1}(w)$,

$$
\begin{array}{cc}
\ln F^{\prime}(w)=\sum_{n=1}^{\infty} B_{n} w^{n}, & \ln K_{1}^{\prime}(w)=\sum_{n=1}^{\infty} b_{n} w^{n}, \\
\Delta(F(w), w)=\sum_{n=0}^{\infty} C_{n} w^{n}, & \Delta\left(K_{1}(w), w\right)=\sum_{n=0}^{\infty} c_{n} w^{n} .
\end{array}
$$

Then $\left|B_{n}\right|<b_{n}$ for $n \in \mathbf{N}$ and $\left|C_{n}\right|<c_{n}$ for $n \in \mathbf{N} \cup\{0\}$. Equality for $n \in \mathbf{N}$ occurs only for the functions $K_{\sigma}(w)=k_{\sigma}^{-1}(w),|\sigma|=1$.

Remarks. In the case of the Schwarzian derivative $\Delta\left(K_{1}(w), w\right)$ we have the simple representation $c_{n}=4^{n} 6(n+1), n \in \mathrm{N} \cup\{0\}$ (see [3]). The first part of the theorem implies Löwner's theorem since each $A_{n}$ is a polynomial with positive coefficients in the $B_{n}{ }^{2}$

Received by the editors November 1, 1979 and, in revised form, January 8, 1980.

1980 Mathematics Subject Classification. Primary 30C50, 30 C75.

Key words and phrases. Univalent functions, Lönner's method.

${ }^{1}$ This research was supported in part by the SFB 40, Theoretische Mathematik, Bonn.

${ }^{2}$ This was pointed out by the referee. 
Proof. The proof follows the same line as the famous proof of Löwner's result (see f.i. [1], [4], [6]). So we need only give here the crucial steps.

If $f \in S, f$ can be embedded into a subordination chain. It results that $F$, the inverse of $f$, has a representation

$$
F(w)=\lim _{t \rightarrow \infty} \Phi\left(e^{-t} w, t\right), \quad \partial \Phi(w, t) / \partial t=w(\partial \Phi(w, t) / \partial w) p(w, t)
$$

with

$p(w, t)=1+\sum_{n=1}^{\infty} p_{n}(t) w^{n}, \quad \operatorname{Re} p(w, t)>0$ for $w \in D, t>0, \Phi(w, 0)=w$.

(For details see [5].)

Using (1) and (2) and setting

$$
\begin{aligned}
& L(w, t):=\ln \frac{\partial \Phi(w, t)}{\partial w}=\sum_{n=0}^{\infty} B_{n}(t) w^{n} \\
& \Delta(w, t):=\Delta(\Phi(w, t), w)=\sum_{n=0}^{\infty} C_{n}(t) w^{n}
\end{aligned}
$$

we get

$$
\begin{aligned}
& \partial L / \partial t=(\partial L / \partial w) w p+(\partial / \partial w)(w p) \\
& \partial \Delta / \partial t=(\partial \Delta / \partial w) w p+2 \Delta(\partial / \partial w)(w p)+\left(\partial^{3} / \partial w^{3}\right)(w p) \\
& B_{0}(t)=t, \quad B_{n}(t)=\int_{0}^{t} e^{n(t-\tau)}\left(\sum_{j=1}^{n-1} j B_{j}(\tau) p_{n-j}(\tau)+(n+1) p_{n}(\tau)\right) d \tau, \quad n \in \mathbf{N}, \\
& C_{n}(t)=\int_{0}^{t} e^{(n+2)(t-\tau)}\left(\sum_{j=0}^{n-1} C_{j}(\tau) p_{n-j}(\tau)(2 n-j+2)+\frac{(n+3) !}{n !} p_{n+2}(\tau)\right) d \tau \\
& n \in \mathbf{N} \cup\{0\}
\end{aligned}
$$

(3) and (4) show that $\operatorname{Re} B_{n}(t)$, resp. $\operatorname{Re} C_{n}(t)$ is maximal for fixed $t$ if and only if we choose $B_{j}(\tau), j=1, \ldots, n-1$, resp. $C_{j}(\tau), j=0, \ldots, n-1, \tau \in[0, t]$ real and maximal and any $p_{j}(\tau)$ involved in (3), resp. (4), equal to the constant 2. As a consequence of (5) and (6) we get that $\operatorname{Max} \operatorname{Re} B_{n}$, resp. $\operatorname{Max} \operatorname{Re} C_{n}, n \in N$, is attained if and only if $p_{1}(t) \equiv 2$ which means $p(w, t)=(1+w) /(1-w)$. Now the assertion of the theorem for $n \in \mathbf{N}$ follows from the fact that the problems of finding the maximum of the real part and the maximum of the modulus for the given coefficients are equivalent (up to a rotation).

The equality $C_{0}=-f^{(3)}(0)+\frac{3}{2}\left(f^{\prime \prime}(0)\right)^{2}$ shows that the remaining case is a classical inequality. 


\section{REFERENCES}

1. W. K. Hayman, Multivalent functions, Cambridge Univ. Press, London and New York, 1958.

2. R. Klouth, Abschätzungen für verallgemeinerte Schwarzsche Derivierte und gewisser Verallgemeinerungen, Dissertation, Bonn. Math. Schr. Nr. 82, 1976.

3. Abschätzungen verallgemeinerter Schwarzscher Derivierter für schlichte holomorphe Funktiomen im Einheitskreis, Preprint des SFB 40 Theoretische Mathematik, Bonn, 1979.

4. K. Löwner, Untersuchungen über schlichte konforme Abbildungen des Einheitskreises. I, Math. Ann. 89 (1923), 103-121.

5. Chr. Pommerenke, Univalent functions, Vandenhoeck und Ruprecht, Göttingen, 1975.

6. G. Schober, Coefficient estimates for inverses of Schlicht functions (preprint).

Mathematisches Institut der Universität, D-53 Bonn, Federal RepUblic of Germany

Mathematisches Institut der Universttät, D-87 WürzbURg, Federal RepUblic of Germany 